\title{
Neural entrainment to the beat in multiple frequency bands in 6-7-
}

\section{year-old children}

\author{
Emese Maróti ${ }^{\mathrm{a}, \mathrm{b}}$, Ferenc Honbolygóo ${ }^{\mathrm{a}, \mathrm{c}}$, Béla Weiss $^{\mathrm{a}}$ \\ ${ }^{a}$ Brain Imaging Centre, Research Centre for Natural Sciences, Hungarian Academy of Sciences, Budapest, Hungary \\ ${ }^{\mathrm{b}}$ Department of Cognitive Science, Budapest University of Technology and Economics, Budapest, Hungary \\ ${ }^{\mathrm{c}}$ Institute of Psychology, Eötvös Loránd University, Budapest, Hungary
}

Manuscript of the article that appeared in:

International Journal of Psychophysiology. 2019; 141:45-55.

DOI: 10.1016/j.ijpsycho.2019.05.005

Entrainment to periodic acoustic stimuli has been found to relate both to the auditory and motor cortices, and it could be influenced by the maturity of these brain regions. However, existing research in this topic provides data about different oscillatory brain activities in different age groups with different musical background. In order to obtain a more coherent picture and examine early manifestations of entrainment, we assessed brain oscillations at multiple time scales (beta: $15-25 \mathrm{~Hz}$, gamma: $28-48 \mathrm{~Hz}$ ) and in steady state evoked potentials (SS-EPs in 
short) in 6-7-year-old children with no musical background right at the start of primary school before they learnt to read. Our goal was to exclude the effect of music training and reading, since previous studies have shown that sensorimotor entrainment (movement synchronization to the beat) is related to musical and reading abilities. We found evidence for endogenous anticipatory processing in the gamma band related to meter perception, and stimulus-related frequency specific responses. However, we did not find evidence for an interaction between auditory and motor networks, which suggests that endogenous mechanisms related to auditory processing may mature earlier than those that underlie motor actions, such as sensorimotor synchronization.

\section{Introduction}

When listening to music we can often feel the urge to move along with the beat. It has been shown that during this time not only our body, but also our brain rhythms synchronize with the music (Large \& Kolen, 1994; Tierney \& Kraus, 2014). Brain rhythms arise due to the simultaneous firing of neural populations (Buzsaki, 2006), and the frequency of these oscillations is related to different perceptual, motor and cognitive processes (Buzsáki \& Draguhn, 2004; MacKay, 1997; Ward, 2003). Previous studies found that neural oscillations can synchronize to an external periodic stimulus via phase and period alignment (Thut, Schyns, \& Gross, 2011), which nevertheless facilitates event processing by allocating attention to the expected periodic time points (Lakatos, Karmos, Mehta, Ulbert, \& Schroeder, 2008; Large \& Jones, 1999; Morillon \& Schroeder, 2015; Schroeder \& Lakatos, 2009; Zion Golumbic et al., 2013). The brain utilizes this entrainment mechanism for speech processing (Ding, Chatterjee, \& Simon, 2014; Ding \& Simon, 2014; Power, Mead, Barnes, \& Goswami, 2012; Power, Mead, Barnes, \& Goswami, 
2013), or when moving to the music (Large, 2010; Nozaradan, Peretz, \& Mouraux, 2012; Tierney \& Kraus, 2014).

Research using periodic auditory stimuli has found entrainment markers in different frequency bands of electroencephalogram (EEG) and magnetoencephalogram (MEG) activity in adults. The phase of the delta band oscillation at target onset predicted reaction time in rhythmic auditory target detection tasks (Stefanics et al., 2010). Delta and theta oscillations entrained to the dominant note rate of musical stimuli that correlated with pitch distortion detection performance (Doelling \& Poeppel, 2015). The time course of the dynamic modulation (synchronization, desynchronization and rebound pattern) of induced beta activity (13-30 Hz) adjusted to stimulus tempo in the auditory cortex (Cirelli et al., 2014; Fujioka, Trainor, Large, \& Ross, 2009), as well as in cortical and subcortical motor areas (Fujioka, Ross, \& Trainor, 2015; Fujioka, Trainor, Large, \& Ross, 2012). Induced gamma activity $(20-60 \mathrm{~Hz})$ predicted tone onsets and persisted when expected tones were omitted (Fujioka et al., 2009; Snyder \& Large, 2005). Induced gamma activity suggests an anticipatory mechanism, which can relate to higherorder processing, such as the perception of metrical hierarchy. Metrical hierarchy refers to the difference between downbeat and other beats within a time unit (called measure), where downbeat is the first beat indicating the beginning of the measure, which gives rise to the perception of pulse in music. Nozaradan and colleagues found evidence for an interaction between sensory- and motor-related neural activities resonating exactly at the stimulus frequency (steady-state evoked potentials, SS-EPs in short) in a form of an additional SS-EP compatible with a nonlinear cross-modulation product of sensorimotor integration. Furthermore, they found phase coupling between auditory and movement related SS-EPs, and selective enhancement of auditory related activities contralateral to the moving hand during tapping. In another study, SS- 
EPs appeared during silent periods after the presentation of auditory rhythms reflecting endogenous neural entrainement (Stupacher, Wood, \& Witte, 2017).

Despite the growing literature on neural entrainment to auditory stimuli, there is very little data regarding children. Although moving to the beat of the music can seem effortless, it is a complex mechanism that takes time to develop (Drake, Jones, \& Baruch, 2000; Eerola, Luck, Toiviainen, \& others, 2006; Fujii et al., 2014; Phillips-Silver \& Trainor, 2005; Repp \& Su, 2013; Zatorre, Chen, \& Penhune, 2007). The earliest markers of entrainment appear in newborn infants suggesting an innate sensitivity to acoustic features that are involved in segregating sound sources already at birth, such as detection of tone onset, offset and change in presentation rate (Háden, Honing, Török, \& Winkler, 2015), functional beat induction (Honing, Ladinig, Háden, \& Winkler, 2009), auditory temporal grouping (Stefanics et al., 2007), and detection of the omission of the downbeat (Winkler, Háden, Ladinig, Sziller, \& Honing, 2009). Infants of 7 and 15 months old showed clear EEG responses frequency locked to the beat and meter frequencies of ambiguous rhythmic patterns (Cirelli, Spinelli, Nozaradan, \& Trainor, 2016). Regarding children, Cirelli and colleagues (2014) found similar tempo dependent fluctuations in induced beta power in 7-year-olds and adults. In children, beta desynchronization was less prominent in the fastest tempo (390 ms inter-onset-interval, in the following: IOI), and in general, there were more variability in children's synchronization than in adults'. Colling and colleagues (2017) found that during passive listening SS-EPs were significantly increased at the stimulus frequency in 9-10-year-old children with dyslexia compared to normally developing children. Conversely, normally developing children showed a cross-modulation SS-EP, whereas children with dyslexia did not. 
Children in these studies were already going to elementary school and have already learnt to read. This is important, because numerous studies found that sensorimotor synchronization, such as tapping to the beat, and reading abilities are related: beat synchronization ability predicted phonological processing, auditory short-term memory and rapid naming (Carr, WhiteSchwoch, Tierney, Strait, \& Kraus, 2014); reading ability increased with decreased tapping variability (Thomson \& Goswami, 2008); the accuracy of tapping out the rhythm of a song predicted spelling ability (Overy, Nicolson, Fawcett, \& Clarke, 2003); meter perception predicted reading accuracy and word reading speed, and meter reproduction predicted pseudoword reading accuracy (Flaugnacco et al., 2014). These studies suggest that the neural correlates of perception and production of structured rhythmic patterns that underlie the segmentation of both speech and music could be responsible for the relationship between sensorimotor synchronization and reading. Another crucial point is that existing studies did not control for the level of musical skills. Music education enhances rhythmic abilities that can facilitate performance on tasks measuring neural entrainment and affect neural responses (Doelling \& Poeppel, 2015; Stupacher et al., 2017). Also, the extent of dynamic coupling that involves cortical and subcortical motor activations and the superior temporal gyrus in the auditory cortex depends on music training, with higher internal coupling in musicians when processing the beat (Chen, Penhune, \& Zatorre, 2009; Grahn \& Rowe, 2009).

In the present study we investigated early developmental manifestations of neural entrainment to periodic acoustic stimuli in children without any musical training before starting elementary school and learning to read. We measured EEG of first-year primary school children while they were either listening passively, or tapping to isochronous tone sequences. Based on previous research, we focused on markers related to tempo dependency, metrical hierarchy and 
interaction between auditory and motor related responses. We were additionally interested in whether there is a relationship between these markers. Importantly, we expected that markers of entrainment found in previous studies may shift in the EEG frequency spectrum as the frequency composition of the EEG power spectra changes throughout childhood and adolescence, which manifests in a decrease in lower frequencies (delta, theta) and a corresponding increase in higher frequencies (alpha, beta and gamma) (Cragg et al., 2011; Ogawa et al., 1984; Takano \& Ogawa, 1998). These changes were suggested to reflect underlying changes in cortico-cortico and cortico-thalamic networks (Lopes da Silva, 1991). Therefore, our analyses focused on spatiotemporal dynamics of evoked and induced oscillations in the 4-60 Hz frequency range, SSEPs and the relationship between these EEG markers.

\section{Methods}

\section{Participants}

Twenty-two right-handed children participated in this study ( 8 girls, mean age: 6.5 years, standard deviation (SD): 0.51 years). Children were recruited from first-year pupils at the Kós Károly Primary School in Budapest. All of them came from middle class families with the same socioeconomic background and none of them received any music training prior to the study. Reading abilities were assessed by the Hungarian version of Dyslexia Differential Diagnosis Maastricht (3DM-H) (Tóth, Csépe, Vaessen, \& Blomert, 2014). Half of the children did not pass the reading control task, and the other half that passed performed 37 percent of the standard level. The study was approved by the United Ethical Review Committee for Research in Psychology (EPKEB, reference number: 2016/062). Parents' written consent and children's assent were obtained. All children had normal hearing, and none of them or their parents 
reported any mental health problems. EEG measurements took place at the Brain Imaging Centre, Research Centre for Natural Sciences, Hungarian Academy of Sciences at the beginning of the first school year before children started to learn to read. Children received a coloring book as a compensation for their participation.

\section{Procedures}

Children completed the entire experiment in a one-hour session. The session comprised of five 10-15-minutes-long measurement blocks with breaks between them that allowed children to rest. During each block children had to perform a different task, and block orders were counterbalanced across children. Three out of the five tasks were linked to entrainment, the other two tasks were linked to pre-attentive processing in speech and music. The latter two were included as part of a longitudinal study, and the related results are to be reported elsewhere. The entrainment related tasks and analyses were adopted from three previous studies (Cirelli et al., 2014; Fujioka et al., 2009; Nozaradan, Zerouali, Peretz, \& Mouraux, 2015) that found distinct neural markers in specific frequency bands and in SS-EPs (detailed description is provided below). We used these tasks in order to be able to reliably compare our results with existing adult or children data. The experimental designs were modified slightly to reduce the length of the EEG session to avoid fatigue in children by using fewer conditions and less repetition within sequences than in the original designs.

\section{Tasks and Stimuli}

\section{Beta task}

We used a similar tone stimulus and experimental design as in (Cirelli et al., 2014; Fujioka et al., 2012). The tone, as well as every stimulus in the study was created in Matlab 8.2.0 
(The Mathworks Inc., Natick, MA, USA) and was presented in PsychToolbox 3 (Brainard, 1997;

Pelli, 1997). The tone was a $262 \mathrm{~Hz}$ pure tone with $10 \mathrm{~ms}$ rise and fall times and $40 \mathrm{~ms}$ steadystate duration. The tone was repeated in isochronous sequences with $390 \mathrm{~ms}, 540 \mathrm{~ms}$ and $780 \mathrm{~ms}$ IOIs, representing three different conditions (Fast, Medium and Slow respectively). The isochronous sequences were presented in seven-seconds-long trains with three-seconds silent time intervals repeated 25 times per condition. Each condition was presented once, and condition order was counterbalanced between subjects. The entire block lasted approximately 12 minutes. Children were listening to the auditory stimuli via Sennheiser PX 200-II on-ear-headphones while watching a silent cartoon of their choice. They were instructed to listen passively and sit still. The aim of the experiment was to examine how children entrain to different tempi, and compare their neural responses in the beta-band to musically trained adults and children with mixed musical background reported in the studies above.

\section{Gamma task}

Auditory stimuli were identical to the omit-loud condition in (Snyder \& Large, 2005). Tones were $262 \mathrm{~Hz}$ pure tones with $10 \mathrm{~ms}$ rise and fall times and $40 \mathrm{~ms}$ steady-state duration. Tones were presented in isochronous sequences with alternating loud-soft accent pattern with $390 \mathrm{~ms}$ IOI. The loud tone was exactly three times louder than the soft tone and loud-soft accent patterns repeated 30 times within one sequence adding up to 60 tones per sequence. There were 30 sequences in the block. The loud tone was missing in one-third of the times. Tone omission was randomized with two constraints: the first two loud-soft accent patterns of the sequence could not contain tone omission, and omission could occur in no more than two subsequent loudsoft accent patterns. Similar to the Beta block, children were listening to the sounds passively while watching a silent cartoon. Our goal was to explore whether and to what extent children 
entrain to the alternating accent pattern before they learn reading and how it compares to adult data (Fujioka et al., 2009; Snyder \& Large, 2005). The significance of the accent pattern is that it gives rise to the perception of metrical hierarchy, which underlies music and linguistic processing. Tone omission, on the contrary, disrupts metrical percept.

\section{Steady-state evoked potentials (SS-EP)}

We replicated the task and tone stimulus in the right hand tapping condition in (Colling et al., 2017; Nozaradan et al., 2015). The stimulus sound was a $33 \mathrm{~s}$ long $333.33 \mathrm{~Hz}$ pure tone amplitude modulated with a $2.4 \mathrm{~Hz}$ periodicity. During the block, the sound was repeated 6 times interspersed with $3 \mathrm{~s}$ silences. Children were asked to tap with the index finger of their right hand on the space key on a computer keyboard to every second beat of the $2.4 \mathrm{~Hz}$ periodicity resulting in a $1.2 \mathrm{~Hz}$ tapping rate. Prior to data recording, children practiced tapping in the presence of the experimenter, until the children reproduced it accurately. The goal of this task was to compare the maturity of neural and sensorimotor synchronization ability in children and examine the interaction between auditory and motor networks. Furthermore, we intended to compare children without musical training to professional adult musicians (Nozaradan et al., 2015) and to older children (Colling et al., 2017).

\section{EEG recording and preprocessing}

EEG data were acquired using BrainAmp amplifiers and an ActiCap active electrode system (BrainProducts GmbH., Munich, Germany). Sixty-four active scalp electrodes were placed according to the 10-10 international electrode system. All channels were referenced to the FCz electrode and impedances were kept below $10 \mathrm{k} \Omega$. Data were sampled at $1000 \mathrm{~Hz}$. 
Preprocessing and data analysis were done in Matlab by using functions from EEGLAB

(Delorme \& Makeig, 2004), ERPLAB (Lopez-Calderon \& Luck, 2014), and custom scripts.

For each of the three tasks, continuous EEG data were bandpass filtered (Butterworth zero-phase filter as implemented in ERPLAB, $0.1 \mathrm{~Hz}-100 \mathrm{~Hz}$ ), and a $50 \mathrm{~Hz}$ notch filter (secondorder IIR notch filter in MATLAB, quality factor $\mathrm{Q}=45$ ) was applied to minimize line-noise artifacts. Eye blinks and eye movements were removed using Independent Component Analysis (Jung et al., 2000) using the runica algorithm (Bell \& Sejnowski, 1995; Makeig et al., 2002). Then, data were segmented according to the given paradigm (details are presented below for each task separately), and for the beta and gamma tasks segments containing artifacts were rejected using EEGLAB with the following criteria: amplitude $([-8080] \mu \mathrm{V})$, slope $(50 \mu \mathrm{V}, \mathrm{R}$ squared limit: 0.5$)$, standard deviation of the mean probability distribution (5), standard deviations from mean kurtosis value (5) and by visual inspection. Finally, surface Laplacian approximation of the scalp current density (SCD) was calculated using the CSD Toolbox (Kayser \& Tenke, 2006; Perrin, Pernier, Bertrand, \& Echallier, 1989) with spline flexibility $m=$ $4, \lambda=10-5$. The advantage of this method is that the Laplacian is a high-pass spatial filter that alleviates the effects of volume conduction in favor of local activity (Nunez \& Srinivasan, 2006).

\section{Data analysis by experimental paradigm}

\section{Beta task}

Continuous data were down-sampled to $250 \mathrm{~Hz}$ and epoched into $1600 \mathrm{~ms}-$ long (-500 ms $1200 \mathrm{~ms}$ relative to tone onset) segments in all three tempo conditions. Next, we calculated timefrequency (TF) representations for each condition from 1 to $60 \mathrm{~Hz}$ in $1 \mathrm{~Hz}$ steps by applying a continuous wavelet transform on each EEG channel using the cmorl-1 complex wavelet function 
in Matlab, which due to its narrow bandwidth $\left(\mathrm{F}_{\mathrm{b}}=1\right)$ and center frequency $\left(\mathrm{F}_{\mathrm{c}}=1\right)$ provides sufficient frequency resolution in higher frequencies, such as in the beta and gamma ranges. Wavelet analyses were done for both evoked and induced activities. For evoked responses, we averaged activity across segments before wavelet analysis. For induced responses, averaged activity was subtracted from each segment, then wavelet was calculated on each segment. Both evoked and induced power spectra were normalized for each frequency for each condition by first subtracting the average power between two subsequent tone onsets, then dividing the resulting power spectra with the average power between two subsequent tone onsets, then multiplying it with 100 so that power spectra were represented in percent change.

In order to locate the auditory cortex, we calculated the mean event-related brain activity for each tempo condition averaged across all subjects (Fig. 1). Subsequent statistical analyses were carried out on those EEG channels where N1 responses were maximal across all subjects (Fig. 1). We used channels only from the left auditory cortex (C5, C3, FC5, FC3). In order to locate significant power changes from baseline in each tempo condition, we averaged power fluctuations in two frequency bands (15-20 Hz and 20-25 Hz) as in (Cirelli et al., 2014), and ran one-sample $t$-tests between two subsequent tone onsets on the average of the four electrodes.

/Insert Figure 1. here/

Figure 1. Group-level event-related EEG activity of the left fronto-central electrode pool (C5, C3, FC5, FC3) for the three tempo conditions (Left). Mean topographical distributions for the latencies between 0 and $150 \mathrm{~ms}$ (top) and between 200 and $300 \mathrm{~ms}$ (bottom) for the Fast condition on all electrodes (Right).

\section{Gamma task}


First, we down-sampled continuous data to $250 \mathrm{~Hz}$, then created epochs from $500 \mathrm{~ms}$ prior the time of the onset of the loud tone (the same when the loud tone was omitted) until 690 ms after the onset of the soft tone resulting in 1190 ms-long segments. Then, similarly to the beta task, a continuous wavelet transform was applied on each EEG channel with center frequencies ranged from 1 to $60 \mathrm{~Hz}$ in $1 \mathrm{~Hz}$ steps, using the same cmorl-1 complex wavelet function. Wavelet was calculated for evoked and induced activities with the same procedure described above. The resulting power spectra were averaged across all subjects and across the left frontocentral electrode pool (C5, C3, FC5, FC3), as in the beta task.

To enable comparisons between the beta and the gamma tasks both evoked and induced power spectra were normalized and represented as percent power change. Next, we derived power values for both the beta $(15-20 \mathrm{~Hz})$ and gamma $(28-48 \mathrm{~Hz})$ frequency ranges and submitted them to repeated-measures ANOVAs, as in (Fujioka et al., 2009).

\section{SS-EP}

For the assessment of SS-EPs, we followed the protocol used in (Nozaradan et al., 2015). We created 6 epochs from the 6 presentations of the sound. We cut the first second of each epoch to remove transient auditory evoked potentials. This shortening did not cut entrainment related activity, because steady beat perception (Repp, 2005) as well as entrainment of SS-EPs (Regan, 1989) require several repetition of the auditory beat. Epochs were then averaged for each subject and transformed in the frequency domain using a discrete Fourier transform, with a $0.031 \mathrm{~Hz}$ frequency resolution. In order to remove background noise, we subtracted from each frequency bin of the FFT amplitude spectrum the average of the neighboring frequency bins ranging from $0.15 \mathrm{~Hz}$ to $-0.09 \mathrm{~Hz}$ and from $+0.09 \mathrm{~Hz}$ to $+0.15 \mathrm{~Hz}$. Because the discrete Fourier transform did not estimate amplitude at the exact frequency of the SS-EP of our interest $(1.2 \mathrm{~Hz}, 2.4 \mathrm{~Hz})$, we 
estimated peak amplitude by averaging the three frequency bins around the given frequency as in (Nozaradan et al., 2015). We additionally estimated peak amplitude for $4.8 \mathrm{~Hz}$, because the frequency spectrum revealed another strong peak at the first harmonic of the frequency of the auditory stimulus. Estimated amplitudes were averaged across all electrodes. Because data were non-normally distributed, we used a Wilcoxon signed-rank test to determine which SS-EP peak is significantly different from zero.

In order to locate SS-EP related oscillations on the scalp, beat- and movement related EEG oscillations were extracted by applying a narrow band Gaussian FFT filter centered at the frequency of interest and $\pm 0.4 \mathrm{~Hz}$ (as the width of the fft window) on the six epochs. The filtered signals were then averaged across epochs and segmented from -0.416 to $+0.416 \mathrm{~ms}$ relative to beat onset. After applying surface Laplacian approximation of the scalp current density (SCD), group-level topographical maps were created at the latency of maximum global field power.

Finally, to assess tapping performance, we calculated the mean error (the difference between the target rate and the individual tapping rate) and standard deviation of the inter-tap intervals, which gave a measurement of accuracy and variability in tapping. In order to exclude accidental double taps or missing taps due to failure to hit the right key, inter-tap intervals smaller than $100 \mathrm{~ms}$ and larger than 1.7 times the tapping period (1416 ms) were removed (3.5\% of the total taps).

\section{Correlations between entrainment markers in the gamma band, SS-EPs and}

\section{tapping variability}


In order to reveal possible relationships between entrainment-related neural responses, we used the power of the gamma frequency band $(28-48 \mathrm{~Hz})$ after tone omission as the representation of the the strength of metrical hierarchy, the amplitude of the $2.4 \mathrm{~Hz}$ SS-EPs and tapping variability, and calculated Spearman's correlation coefficients. Outliers were removed pairwise.

\section{Results}

\section{Beta task}

Fig. 2 shows time-frequency representations of evoked and induced beta-band activity for the three tempo conditions. The display was reduced to the beat window from tone onset to the subsequent tone onset (as we used this window for analyses), and 4-60 Hz frequency range.

/Insert Figure 2. here/

Figure 2. Group-level time-frequency representations for evoked and induced power $(4-60 \mathrm{~Hz})$ in the three tempo conditions (390 ms, $540 \mathrm{~ms}, 780 \mathrm{~ms}$ from left to right), averaged for four electrodes (C5, C3, FC5, FC3). Values are represented as percent power changes. Each plot spans one beat window from tone onset to tone onset.

Induced power fluctuations are shown in Fig. 3 for the low $(15-20 \mathrm{~Hz})$ and high $(20-25 \mathrm{~Hz})$ beta frequency ranges. Induced beta activity showed a pattern of synchronization after tone onset followed by a desynchronization. Contrary to previous adult and child data (Cirelli et al., 2014), there is no clear rebound (positive values before the onset of the next tone), except in the Slow tempo condition in the high beta range. We ran one-sample $t$-tests to determine the time intervals during which power change differed significantly from zero, and applied Bonferroni-Holm 
correction for multiple comparisons. No power changes were significant at 0.05 alpha level, therefore we did not conduct any further analyses regarding this task.

/Insert Figure 3. here/

Figure 3. Induced power fluctuations in the low $(15-20 \mathrm{~Hz})$ and high $(20-25 \mathrm{~Hz})$ beta ranges averaged for four electrodes (C5, C3, FC5, FC3). Significant power changes were determined by one-sample $t$ tests (Yellow, $\mathrm{p}<0.1$ (Bonferroni-Holm)).

\section{Gamma task}

Fig. 4 shows group-level time-frequency representations of evoked and induced power $(4-60 \mathrm{~Hz})$ for the two conditions (omit and standard). Evoked activity decreased after the omission of the loud tone compared to the standard condition where the loud tone was present. Regarding induced activity, beta $(15-20 \mathrm{~Hz})$ and gamma $(28-48 \mathrm{~Hz})$ power showed different patterns of power changes throughout the conditions. There was a considerable increase in beta power around $100 \mathrm{~ms}$ followed by a decrease around $300 \mathrm{~ms}$ in the standard condition, then power was gradually rising until the onset of the soft tone. In the omit condition beta power increased slighly around $100 \mathrm{~ms}$, then decreased around $200 \mathrm{~ms}$ and returned near the value of the previous increase before $300 \mathrm{~ms}$. Then, power stayed around the same value until the onset of the soft tone with a slight dip right before tone onset. On the contrary, gamma power increased around 60 ms in both conditions regardless of whether the loud tone was present or absent (Fig. 5).

In order to assess the differences in power changes in the beta band between conditions, power values of beta band activity were entered into a repeated-measures ANOVA with factors condition (omit vs. standard) and latency (100 ms vs. $300 \mathrm{~ms}$ ). Induced power in the standard condition differed considerably between the two latencies $\left(\mathrm{M}_{100}=2.19, \mathrm{SD}=3, \mathrm{M}_{300}=-1.88\right.$, 
$\mathrm{SD}=2.95)$, as opposed to the power in the omit condition $\left(\mathrm{M}_{100}=0.77, \mathrm{SD}=6.45, \mathrm{M}_{300}=1.04\right.$, $\mathrm{SD}=10)$. However, none of the main effects and interaction were significant. We also compared the power of gamma band activity between conditions. Amplitudes at $60 \mathrm{~ms}$ and at half-time of the beat preceding the onset of the time of the loud tone $(-195 \mathrm{~ms})$ were entered into a repeatedmeasures ANOVA with factors condition (omit vs. standard) and latency (60 ms vs. -195 ms). There was a significant main effect of latency $F(1,21)=4.44, p=0.047, \eta^{2}=0.175$. Power was significantly higher at $60 \mathrm{~ms}$ after tone onset or omission, than at half-time of the beat prior to the time of the loud tone $\left(\mathrm{M}_{60}=2.14, \mathrm{SD}=3.42, \mathrm{M}_{-195}=-0.63, \mathrm{SD}=4.57\right)$. The main effect of condition and the interaction were not significant, suggesting that gamma activity was not affected by the presence or absence of the loud tone.

/Insert Figure 4. here/

Figure 4. Group-level time-frequency representations of evoked and induced power $(4-60 \mathrm{~Hz})$ for the omit and standard conditions, averaged for four electrodes (C5, C3, FC5, FC3). Values are represented as percent power changes. Each plot contains two subsequent tone onsets (loud tone at $0 \mathrm{~ms}$, and soft tone at $390 \mathrm{~ms}$ ).

/Insert Figure 5. here/

Figure 5. Induced power fluctuations in the beta $(15-20 \mathrm{~Hz})$ and gamma $(28-48 \mathrm{~Hz})$ ranges averaged for four electrodes (C5, C3, FC5, FC3) for the omit and standard conditions.

\section{SS-EP}

One participant's neural responses were beyond three times the inter-quartile-range, therefore it was removed as an outlier from the plots and from the statistical analyses. The frequency spectrum (Fig. 6, bottom) revealed two prominent SS-EP peaks at $2.4 \mathrm{~Hz}$ and at 4.8 
$\mathrm{Hz}$, and somewhat smaller peaks at $0.8 \mathrm{~Hz}, 1.2 \mathrm{~Hz}$ and 1.6 Hz. CSD topographical maps relative to beat onset (Fig. 6, top left) confirmed that the $2.4 \mathrm{~Hz}$ and the $4.8 \mathrm{~Hz}$ SS-EP peaks were related to the auditory stimuli (the $4.8 \mathrm{~Hz}$ being the first upper harmonic of the beat frequency).

However, the $0.8 \mathrm{~Hz}, 1.2 \mathrm{~Hz}$ and $1.6 \mathrm{~Hz}$ activities did not show a clear connection either to auditory or motor brain areas. While the $1.2 \mathrm{~Hz}$ is the target rate of tapping, the 0.8 and $1.6 \mathrm{~Hz}$ frequencies correspond to the third of the beat rate $(2.4 / 3=0.8 \mathrm{~Hz})$ and its first upper harmonic $(0.8 * 2=1.6 \mathrm{~Hz})$. Wilcoxon signed-rank tests confirmed that the two stimulus-related peaks were significantly different from zero $(2.4 \mathrm{~Hz}: \mathrm{Z}=4.01, \mathrm{p}<0.001,4.8 \mathrm{~Hz}: \mathrm{Z}=3.94, \mathrm{p}<0.001)$. However, the other SS-EPs were not significant.

/Insert Figure 6. here/

Figure 6. Group-level average frequency spectrum of the noise-subtracted EEG signals averaged across all channels (bottom) with CSD topographical maps relative to beat onset (top left) and relative to the tapping onset (top right) corresponding to the $0.8,1.2,1.6,2.4$ and $4.8 \mathrm{~Hz} \mathrm{SS}-\mathrm{EP}$ peaks, at the latency of maximum global field power.

The group-level mean tapping error was -0.027 seconds with 0.14 seconds average tapping variability, which indicated that children tapped slightly faster than the target rate 1.2 Hz. However, the distribution of tapping frequencies (Fig. 7) revealed that almost 24 percent of the children tapped at a rate that corresponds to the third of the beat rate or its first upper harmonic. Since topographical maps of $0.8,1.2$ and $1.6 \mathrm{~Hz}$ did not show a clear connection to motor areas, and tapping frequencies suggest that tapping rate was highly variable between children, we created topographical maps relative to the actual tapping onsets (Fig. 6 top right). The maps revealed motor related activations for the $0.8,1.2$ and $1.6 \mathrm{~Hz}$ SS-EPs. 
/Insert Figure 7. here/

Figure 7. The distribution of tapping frequencies.

\section{Correlations between entrainment markers in the gamma band, SS-EPs and} tapping variability

There was only one correlation between tapping variability and the amplitude of the 2.4 Hz SS-EP $\left(r_{s}=-0.46\right.$, puncorrected $\left.=0.035\right)$, as shown in Fig. 8. Lower levels of tapping variability was associated with larger amplitudes. However, after correcting for multiple comparisons the relationship was no longer significant.

/Insert Figure 8. here/

Figure 8. Scatter plot of the tapping variability and amplitude of the $2.4 \mathrm{~Hz}$ SS-EP. Lower levels of tapping variability was associated with larger amplitudes.

\section{Discussion}

In this study, we explored early markers of neural entrainment to periodic auditory stimuli for children without formal musical training before starting elementary school and learning to read. Because entrainment, especially sensorimotor entrainment (the ability to synchronize movement to a periodic stimulus), has been linked to various musical and linguistic skills and attention (Carr et al., 2014; Chen, Penhune, \& Zatorre, 2008; Colling et al., 2017; Maróti et al., 2018; Repp, 2010; Tierney \& Kraus, 2013) our primary goal was to exclude any influence of training or education that might enhance these skills. Present study nevertheless aimed to compare neural responses of 6-7-year-olds' to existing data that include adults, subjects with musical training and older children. Comparisons were made primarily with three previous 
studies, from which we adopted our tasks and analyses (Cirelli et al., 2014; Fujioka et al., 2009; Nozaradan et al., 2015). Finally, we examined the relationship between entrainment related neural responses across tasks to better understand how mechanisms related to different auditory and motor processing might be related.

\section{Beta task}

First, analyses were made in the beta band (15-25 Hz). Similarly to existing literature (Cirelli et al., 2014; Fujioka, Trainor, Large, \& Ross, 2009; Fujioka et al., 2012), induced beta activity showed a pattern of synchronization after tone onset followed by a desynchronization. Contrary to previous child and adult data, there was no clear anticipatory rebound and power changes did not differ significantly from zero. Beta rebound was proposed to reflect an endogenous anticipatory timing mechanism for isochronous tone sequences (Fujioka et al., 2012), and it has been related to motor control (Gilbertson et al., 2005; Pogosyan, Gaynor, Eusebio, \& Brown, 2009) and cortical network reset (Pfurtscheller, Müller-Putz, Pfurtscheller, \& Rupp, 2005). This suggests a possible role of the auditory system to guide movements for predictive time intervals. The absence of the anticipatory rebound in 6-7-year-olds might indicate that this process has not completely developed yet. Alternatively, it is possible that beta oscillations were less coherent. This possibility is supported by the large group variability in the latency of the desynchronization in all tempo conditions for both frequency ranges (High beta: $\mathrm{SD}_{\text {Fast }}=108.75 \mathrm{~ms}, \mathrm{SD}_{\text {Medium }}=163.1 \mathrm{~ms}, \mathrm{SD}_{\text {Slow }}=216.7 \mathrm{~ms}$, Low beta: $\mathrm{SD}_{\text {Fast }}=85.85 \mathrm{~ms}$, $\mathrm{SD}_{\text {Medium }}=124.09 \mathrm{~ms}, \mathrm{SD}_{\text {Slow }}=155.32 \mathrm{~ms}$ ). Cirelli and colleagues found similarly that the time course of desynchronization was highly variable in children, and the dynamic fluctuation pattern was weaker in children compared to adults (Cirelli et al., 2014). The reason why their subjects exhibited anticipatory responses could be due to the fact that half of the children and all adults 
received formal musical training. Music training was found to enhance auditory processing (Koelsch, Schröger, \& Tervaniemi, 1999; Kraus \& Chandrasekaran, 2010; Musacchia, Sams, Skoe, \& Kraus, 2007; Schlaug, Norton, Overy, \& Winner, 2005; Tallal \& Gaab, 2006). Because of the high variability in the latency of desynchronization in both Cirelli and colleagues' and our study, it is more likely that the consistency of beta oscillations have less effect on the predictive timing mechanism. Rather, it is possible that the underlying neural mechanisms mature earlier in children that receive music training.

\section{Gamma task}

Next, we examined gamma activity $(28-48 \mathrm{~Hz})$ relative to beta activity $(15-20 \mathrm{~Hz})$ during tone omissions. Similarly to Fujioka and colleagues (2009), the fluctuation of induced gamma as opposed to induced beta activity was uninterrupted after tone omission. This was apparent in the difference between the standard and omit conditions. In the standard condition, there was a substantial increase and a decrease in beta power after the onset of the loud tone, whereas in the omit condition, a small initial increase was followed by a brief decrease, then power returned to the previous increased level. Fujioka and colleagues (2009) proposed that the decrease in induced auditory beta activity facilitates motor preparatory processes for expected tones in a sequence that aid movement synchronization to acoustic signals. When these tones are occasionally omitted, the regular pattern is interrupted, therefore the preparatory process becomes perturbed. It seems in our child data that the small initial increase and decrease after tone omissions are a relfection of the perturbation in the preparatory process to the next tone. In Fujioka's study, adults seem to adapt better to the occasional omissions, as the motor preparatory decrease is absent, and the initial rising of beta power continues until the onset of the next tone. Because the decrease did not disappear in children, only lessened, it could explain why the 
difference between the two conditions was not significant. Induced gamma activity on the contrary is involved in encoding the periodicity of the stimulus, which facilitates entrainment through internal anticipatory processes. Our child data is consistent with previous literature including adults with extensive musical training (Fujioka et al., 2009; Snyder \& Large, 2005), indicating an adultlike endogenous anticipatory processing in the gamma band in children. One of the strongest predictors of metrical percept (Hannon, Snyder, Eerola, \& Krumhansl, 2004) and maintenance of metrical representation (Large, Fink, \& Kelso, 2002) is temporal accent. Since induced activity was preserved even after the omission of the accented note (loud tone), it suggests that 6-7-year-old children, similarly to adults (Fujioka et al., 2009; Snyder \& Large, 2005) perceive metrical structure in accented rhythmic sequences. This is not surprising, since previous studies found that infants are already capable of detecting the omission of the downbeat (Winkler et al., 2009), and they show EEG responses frequency locked to the beat and meter frequencies of ambiguous rhythmic patterns (Cirelli et al., 2016).

\section{SS-EP}

SS-EPs have been previously shown to reflect not only sensory processes but interaction between modalities, such as dynamic coupling between auditory and motor cortices in the case of synchronized tapping to the beat (Nozaradan et al., 2015). Our analyses of SS-EPs revealed peaks at the exact stimulus frequency $(2.4 \mathrm{~Hz})$ and at its first harmonic $(4.8 \mathrm{~Hz})$ that seemed to correspond to auditory regions of the brain. Furthermore, there were other, non-significant peaks at the tapping frequency $(1.2 \mathrm{~Hz})$, at the third of the beat frequency $(0.8 \mathrm{~Hz})$ and at its first upper harmonic $(1.6 \mathrm{~Hz})$ that related to motor areas in the brain. In the study of Nozaradan and colleagues (2011), participants showed SS-EPs at the half and third of the beat frequency and their upper harmonics corresponding to binary and ternary metric levels of the beat when 
instructed to imagine the given metric structure. The perception of a given metric structure introduces additional periodicities corresponding to integer ratios of the beat frequency, and it was shown that humans have a natural preference for those integer ratios in timing perception and production (Brochard, Abecasis, Potter, Ragot, \& Drake, 2003; Essens, 1986; Repp, 2005). Despite that children were not instructed to imagine a certain metric structure while tapping (although tapping to every second beat would imply a binary metric structure), perception of different metric structures (binary and ternary) in our data was indicated both in the EEG spectrum and in the tapping performance.

Contrary to adult musicians and older children, there was no cross-modulation SS-EP peak at $3.6 \mathrm{~Hz}(1.2+2.4 \mathrm{~Hz})$, which was considered by Nozaradan and colleagues (2015) the proof of auditory-motor interaction that drives sensorimotor entrainment. A possible reason for the lack of the cross-modulation SS-EP could be that tapping rate was not consistent across children as some tapped in a binary meter (target rate), some tapped in a ternary meter, or at the rate of the first harmonic of the ternary meter. This inconsistency led to the fact that motor activity was not visible on the topographical maps related to the beat onset, and only appeared when maps were created relative to the actual tapping onsets. Sensorimotor synchronization ability constantly matures during childhood, and starts to reach adult level around the age of 8-9 (Repp \& Su, 2013). Therefore, it is possible that children at the age of 6-7 are not fully capable of reproducing a required timing rate and they deviate towards their preferred metrical representation. Conversely, 10-year-old typically developing children were already able to reproduce the $1.2 \mathrm{~Hz}$ target rate (with a $2.4 \mathrm{~Hz}$ beat frequency) and their EEG displayed a significant SS-EP at $3.6 \mathrm{~Hz}$ compared to matched aged children with dyslexia (Colling et al., 2017). Also, music training has been found to be related to the extent of dynamic coupling as 
well, with higher internal coupling in musically trained subjects (Chen et al., 2009; Grahn \& Rowe, 2009). Previous studies that found a significant cross-modulation SS-EP in adults tested only musicians (Nozaradan et al., 2011, 2012, 2015). If the typically developing children in the study of Colling and colleagues (2017) received music training (there is no information about it), one can conceive that the cross-modulation SS-EP emerges with musical expertise.

\section{Correlations between entrainment markers in the gamma band, SS-EPs and} tapping variability

In order to reveal possible relationships between entrainment-related neural activities, we compared neural responses in the gamma band, SS-EP and tapping variability. We found a relationship between tapping variability and the amplitude of the SS-EP related to the frequency of the auditory stimulus. This is not surprising, given that sensorimotor synchronization requires perceptual processing of the auditory stimulus, which guides motor actions (Repp \& Su, 2013). However, after correcting for multiple comparisons the correlation was no longer significant.

\section{Limitations}

Our study's major limitation is the fewer number of trials compared to previous studies using the same paradigms. For the beta task, Cirelli and colleagues (2014) repeated each condition twice in the experiment, resulting in about twice as many trials as ours giving more statistical power. For the gamma task, Fujioka and colleagues (2009) had an extra condition, where the soft tone was occasionally omitted, and neural responses were averaged across tones and across omissions. Finally in the SS-EP task, we used only the tapping with right hand condition out of the three conditions including listening only, tapping with right hand and tapping with left hand (Nozaradan et al., 2015). 
Another factor that it is worth noting is the tempo range of our stimuli. McAuley and colleagues (2006) found that people throughout their lifespan have different age-specific entrainment regions. That is, the range of periods that they can synchronize most effectively to. The width of the entrainment region is smaller in childhood than in adulthood. In fact, there are significant differences between age groups in childhood. The authors found a sudden increase in entrainment region at the age of 8-9 compared to the previous age groups. Importantly, the greater the distance from the entrainment region, the greater the variability in sensorimotor synchronization and produced periods drift toward the age-specific entrainment region. The entrainment region is characterized by the peak of spontaneous motor tempo, which is the preferred period of unpaced tapping. For children at age 6-7, the peak of spontaneous motor tempo is $200-300 \mathrm{~ms}$. In our study, the fastest stimulus tempo was $390 \mathrm{~ms}$, therefore it is possible that slow stimulus tempi resulted in greater variability in the neural responses. This prevailed in the increase in the variability of the latency of beta desynchronization towards the slower tempi, and the different tapping rates in the sensorimotor synchronization task.

In the future, we plan to investigate the developmental trajectory of these neural responses and shed light on possible relationships between underlying neural processes by following children throughout the first four years of primary school and measuring their neural entrainment each year. Additionally, we plan to investigate the effect of music training on the development on these neural entrainment markers and compare groups with and without musical background. Finally, we would like to compare markers of neural entrainment to behavioral data, such as reading, rhythmic skills, or sensorimotor synchronization ability.

\section{Conclusions}


Entrainment to periodic acoustic stimuli has been found to relate both to auditory and motor processes and it seems to be influenced by the maturity of the underlying neural networks during development. Induced beta oscillations in the auditory cortex have been proposed to facilitate motor preparatory processes by providing timing cues driven by the stimulus, while gamma oscillations reflect internal anticipatory timing (Fujioka et al., 2009) that nevertheless gives rise to metrical percept (Snyder \& Large, 2005). During sensorimotor synchronization, auditory-motor interactions manifest in a non-linear product of auditory- and motor related SSEPs (Nozaradan et al., 2015). Our findings suggest that in 6-7-year-old children without music training and before going to school and learning to read, entrainment related neural responses associated with different frequency oscillations do not develop at the same rate. Endogenous mechanisms that do not require motor actions, such as the predictive induced gamma activity related to metrical perception seems to mature earlier than processes that underlie movement, such as sensorimotor synchronization or movement preparation. A long line of studies found that sensorimotor synchronization takes many years until it reaches adult level (for a review, see Repp \& Su, 2013), while metrical percept seems to be present already at infancy (Háden et al., 2015; Stefanics et al., 2007; Winkler et al., 2009). The maturation of movement related processes might have a protracted trajectory. Whether it is affected by the improvement of rhythmic skills related to education, or it is part of natural development remains to be investigated.

\section{Acknowledgment}

The study was supported by the Subject Pedagogy Research Program of the Hungarian Academy of Sciences (SZ-009/2016) and by the János Bolyai research fellowship awarded to Ferenc Honbolygó. We would like to thank the members of the MTA-LFZE Research Group on Active Music Learning, László Nemes, Gabriella Deszpot, Edina Barabás, Borbála Szirányi, and 
Tamara Farnadi for their help in the development of the theorethical concepts of the research.

We are thankful to Borbála Lukács, Fanni Eckhardt, Gabriella Baliga, Borbála German, Eszter Tóth-Fáber, Borbála Tölgyesi, Dóra Szabó, Anna Fekete, Zsuzsanna Lakatos, and María de Lourdes Noboa Cepeda for their help in EEG data recording. We thank the teachers of Kós Károly Primary School for their assistance in the study, and the pupils of the school for their participation.

\section{References}

Bell, A. J., \& Sejnowski, T. J. (1995). An information-maximization approach to blind separation and blind deconvolution. Neural Computation, 7(6), 1129-1159.

Brainard, D. H. (1997). The psychophysics toolbox. Spatial Vision, 10, 433-436.

Brochard, R., Abecasis, D., Potter, D., Ragot, R., \& Drake, C. (2003). The "Ticktock" of Our Internal Clock: Direct Brain Evidence of Subjective Accents in Isochronous Sequences. Psychological Science, 14(4), 362-366. https://doi.org/10.1111/1467-9280.24441

Buzsaki, G. (2006). Rhythms of the Brain. Oxford University Press. Retrieved from https://www.google.com/books?hl=en\&|r=\&id=ldz58irprjYC\&oi=fnd\&pg=PA4\&dq=brain+rhyth ms+buzsaki\&ots=Q2S622hOJP\&sig=GzCpb_AIJUaJKlyzw3pF0KhEh5g

Buzsáki, G., \& Draguhn, A. (2004). Neuronal oscillations in cortical networks. Science, 304(5679), 19261929.

Carr, K. W., White-Schwoch, T., Tierney, A. T., Strait, D. L., \& Kraus, N. (2014). Beat synchronization predicts neural speech encoding and reading readiness in preschoolers. Proceedings of the National Academy of Sciences, 111(40), 14559-14564. 
Chen, J. L., Penhune, V. B., \& Zatorre, R. J. (2008). Listening to Musical Rhythms Recruits Motor Regions of the Brain. Cerebral Cortex, 18(12), 2844-2854. https://doi.org/10.1093/cercor/bhn042

Chen, J. L., Penhune, V. B., \& Zatorre, R. J. (2009). The Role of Auditory and Premotor Cortex in Sensorimotor Transformations. Annals of the New York Academy of Sciences, 1169(1), 15-34. https://doi.org/10.1111/j.1749-6632.2009.04556.x

Cirelli, L. K., Bosnyak, D., Manning, F. C., Spinelli, C., Marie, C., Fujioka, T., ... Trainor, L. J. (2014). Beatinduced fluctuations in auditory cortical beta-band activity: using EEG to measure age-related changes. Frontiers in Psychology, 5. https://doi.org/10.3389/fpsyg.2014.00742

Cirelli, L. K., Spinelli, C., Nozaradan, S., \& Trainor, L. J. (2016). Measuring Neural Entrainment to Beat and Meter in Infants: Effects of Music Background. Frontiers in Neuroscience, 10. https://doi.org/10.3389/fnins.2016.00229

Colling, L. J., Noble, H. L., \& Goswami, U. (2017). Neural entrainment and sensorimotor synchronization to the beat in children with developmental dyslexia: An EEG study. Frontiers in Neuroscience, 11. Retrieved from https://www.ncbi.nlm.nih.gov/pmc/articles/PMC5506338/

Cragg, L., Kovacevic, N., McIntosh, A. R., Poulsen, C., Martinu, K., Leonard, G., \& Paus, T. (2011). Maturation of EEG power spectra in early adolescence: a longitudinal study. Developmental Science, 14(5), 935-943. https://doi.org/10.1111/j.1467-7687.2010.01031.x

Delorme, A., \& Makeig, S. (2004). EEGLAB: an open source toolbox for analysis of single-trial EEG dynamics including independent component analysis. Journal of Neuroscience Methods, 134(1), 9-21.

Ding, N., Chatterjee, M., \& Simon, J. Z. (2014). Robust cortical entrainment to the speech envelope relies on the spectro-temporal fine structure. Neuroimage, $88,41-46$. 
Ding, N., \& Simon, J. Z. (2014). Cortical entrainment to continuous speech: functional roles and interpretations. Frontiers in Human Neuroscience, 8. Retrieved from https://www.ncbi.nlm.nih.gov/pmc/articles/PMC4036061/

Doelling, K. B., \& Poeppel, D. (2015). Cortical entrainment to music and its modulation by expertise. Proceedings of the National Academy of Sciences, 112(45), E6233-E6242. https://doi.org/10.1073/pnas.1508431112

Drake, C., Jones, M. R., \& Baruch, C. (2000). The development of rhythmic attending in auditory sequences: attunement, referent period, focal attending. Cognition, 77(3), 251-288.

Eerola, T., Luck, G., Toiviainen, P., \& others. (2006). An investigation of pre-schoolers' corporeal synchronization with music. In Proceedings of the 9th international conference on music perception and cognition (pp. 472-476). The Society for Music Perception and Cognition and European Society for the Cognitive Sciences of Music Bologna. Retrieved from http://www.academia.edu/download/3444610/235.pdf

Essens, P. J. (1986). Hierarchical organization of temporal patterns. Perception \& Psychophysics, 40(2), 69-73. https://doi.org/10.3758/BF03208185

Flaugnacco, E., Lopez, L., Terribili, C., Zoia, S., Buda, S., Tilli, S., ... others. (2014). Rhythm perception and production predict reading abilities in developmental dyslexia. Frontiers in Human Neuroscience, 8. Retrieved from https://www.ncbi.nlm.nih.gov/pmc/articles/PMC4045153/

Fujii, S., Watanabe, H., Oohashi, H., Hirashima, M., Nozaki, D., \& Taga, G. (2014). Precursors of dancing and singing to music in three-to four-months-old infants. PloS One, 9(5), e97680.

Fujioka, T., Ross, B., \& Trainor, L. J. (2015). Beta-Band Oscillations Represent Auditory Beat and Its Metrical Hierarchy in Perception and Imagery. The Journal of Neuroscience : The Official Journal of the Society for Neuroscience, 35(45), 15187. 
Fujioka, T., Trainor, L. J., Large, E. W., \& Ross, B. (2009). Beta and gamma rhythms in human auditory cortex during musical beat processing. Annals of the New York Academy of Sciences, 1169(1), 89-92.

Fujioka, T., Trainor, L. J., Large, E. W., \& Ross, B. (2012). Internalized timing of isochronous sounds is represented in neuromagnetic beta oscillations. Journal of Neuroscience, 32(5), 1791-1802.

Gilbertson, T., Lalo, E., Doyle, L., Di Lazzaro, V., Cioni, B., \& Brown, P. (2005). Existing motor state is favored at the expense of new movement during $13-35 \mathrm{~Hz}$ oscillatory synchrony in the human corticospinal system. Journal of Neuroscience, 25(34), 7771-7779.

Grahn, J. A., \& Rowe, J. B. (2009). Feeling the Beat: Premotor and Striatal Interactions in Musicians and Nonmusicians during Beat Perception. Journal of Neuroscience, 29(23), 7540-7548. https://doi.org/10.1523/JNEUROSCI.2018-08.2009

Habibi, A., Cahn, B. R., Damasio, A., \& Damasio, H. (2016). Neural correlates of accelerated auditory processing in children engaged in music training. Developmental Cognitive Neuroscience, 21, 114.

Háden, G. P., Honing, H., Török, M., \& Winkler, I. (2015). Detecting the temporal structure of sound sequences in newborn infants. International Journal of Psychophysiology, 96(1), 23-28. https://doi.org/10.1016/j.ijpsycho.2015.02.024

Hannon, E. E., Snyder, J. S., Eerola, T., \& Krumhansl, C. L. (2004). The role of melodic and temporal cues in perceiving musical meter. Journal of Experimental Psychology: Human Perception and Performance, 30(5), 956.

Honing, H., Ladinig, O., Háden, G. P., \& Winkler, I. (2009). Is Beat Induction Innate or Learned? Annals of the New York Academy of Sciences, 1169(1), 93-96. https://doi.org/10.1111/j.17496632.2009.04761.x 
Jung, T.-P., Makeig, S., Westerfield, M., Townsend, J., Courchesne, E., \& Sejnowski, T. J. (2000). Removal of eye activity artifacts from visual event-related potentials in normal and clinical subjects. Clinical Neurophysiology, 111(10), 1745-1758.

Kayser, J., \& Tenke, C. E. (2006a). Principal components analysis of Laplacian waveforms as a generic method for identifying ERP generator patterns: I. Evaluation with auditory oddball tasks. Clinical Neurophysiology, 117(2), 348-368.

Kayser, J., \& Tenke, C. E. (2006b). Principal components analysis of Laplacian waveforms as a generic method for identifying ERP generator patterns: I. Evaluation with auditory oddball tasks. Clinical Neurophysiology, 117(2), 348-368.

Koelsch, S., Schröger, E., \& Tervaniemi, M. (1999). Superior pre-attentive auditory processing in musicians. Neuroreport, 10(6), 1309-1313.

Kraus, N., \& Chandrasekaran, B. (2010). Music training for the development of auditory skills. Nature Reviews. Neuroscience, 11(8), 599.

Lakatos, P., Karmos, G., Mehta, A. D., Ulbert, I., \& Schroeder, C. E. (2008). Entrainment of neuronal oscillations as a mechanism of attentional selection. Science, 320(5872), 110-113.

Large, E. W. (2010). Neurodynamics of music. In Music perception (pp. 201-231). Springer. Retrieved from http://link.springer.com/10.1007/978-1-4419-6114-3_7

Large, E. W., Fink, P., \& Kelso, S. J. (2002). Tracking simple and complex sequences. Psychological Research, 66(1), 3-17.

Large, E. W., \& Jones, M. R. (1999). The dynamics of attending: How people track time-varying events. Psychological Review, 106(1), 119-159. https://doi.org/10.1037/0033-295X.106.1.119

Large, E. W., \& Kolen, J. F. (1994). Resonance and the perception of musical meter. Connection Science, $6(2-3), 177-208$. 
Lopes da Silva, F. (1991). Neural mechanisms underlying brain waves: from neural membranes to networks. Electroencephalography and Clinical Neurophysiology, 79(2), 81-93. https://doi.org/10.1016/0013-4694(91)90044-5

Lopez-Calderon, J., \& Luck, S. J. (2014). ERPLAB: an open-source toolbox for the analysis of event-related potentials. Frontiers in Human Neuroscience, 8. Retrieved from https://www.ncbi.nlm.nih.gov/pmc/articles/PMC3995046/

MacKay, W. A. (1997). Synchronized neuronal oscillations and their role in motor processes. Trends in Cognitive Sciences, 1(5), 176-183.

Makeig, S., Westerfield, M., Jung, T.-P., Enghoff, S., Townsend, J., Courchesne, E., \& Sejnowski, T. J. (2002). Dynamic brain sources of visual evoked responses. Science, 295(5555), 690-694.

Maróti, E., Barabás, E., Deszpot, G., Farnadi, T., Nemes, L. N., Szirányi, B., \& Honbolygó, F. (2018). Does moving to the music make you smarter? The relation of sensorimotor entrainment to cognitive, linguistic, musical, and social skills: Psychology of Music.

https://doi.org/10.1177/0305735618778765

McAuley, J. D., Jones, M. R., Holub, S., Johnston, H. M., \& Miller, N. S. (2006). The time of our lives: life span development of timing and event tracking. Journal of Experimental Psychology: General, 135(3), 348.

Morillon, B., \& Schroeder, C. E. (2015). Neuronal oscillations as a mechanistic substrate of auditory temporal prediction. Annals of the New York Academy of Sciences, 1337(1), 26-31. https://doi.org/10.1111/nyas.12629

Musacchia, G., Sams, M., Skoe, E., \& Kraus, N. (2007). Musicians have enhanced subcortical auditory and audiovisual processing of speech and music. Proceedings of the National Academy of Sciences, 104(40), 15894-15898. 
Nozaradan, S., Peretz, I., Missal, M., \& Mouraux, A. (2011). Tagging the neuronal entrainment to beat and meter. Journal of Neuroscience, 31(28), 10234-10240.

Nozaradan, S., Peretz, I., \& Mouraux, A. (2012). Selective neuronal entrainment to the beat and meter embedded in a musical rhythm. The Journal of Neuroscience : The Official Journal of the Society for Neuroscience, 32(49), 17572.

Nozaradan, S., Zerouali, Y., Peretz, I., \& Mouraux, A. (2015). Capturing with EEG the Neural Entrainment and Coupling Underlying Sensorimotor Synchronization to the Beat. Cerebral Cortex, 25(3), 736747. https://doi.org/10.1093/cercor/bht261

Nunez, P. L., \& Srinivasan, R. (2006). Electric fields of the brain: the neurophysics of EEG. Oxford University Press, USA.

Ogawa, T., Sugiyama, A., Ishiwa, S., Suzuki, M., Ishihara, T., \& Sato, K. (1984). Ontogenic development of autoregressive component waves of waking EEG in normal infants and children. Brain and Development, 6(3), 289-303. https://doi.org/10.1016/S0387-7604(84)80042-X

Overy, K., Nicolson, R. I., Fawcett, A. J., \& Clarke, E. F. (2003). Dyslexia and music: measuring musical timing skills. Dyslexia, 9(1), 18-36.

Pelli, D. G. (1997). The VideoToolbox software for visual psychophysics: Transforming numbers into movies. Spatial Vision, 10(4), 437-442.

Perrin, F., Pernier, J., Bertrand, O., \& Echallier, J. F. (1989a). Spherical splines for scalp potential and current density mapping. Electroencephalography and Clinical Neurophysiology, 72(2), 184-187.

Perrin, F., Pernier, J., Bertrand, O., \& Echallier, J. F. (1989b). Spherical splines for scalp potential and current density mapping. Electroencephalography and Clinical Neurophysiology, 72(2), 184-187.

Pfurtscheller, G., Müller-Putz, G. R., Pfurtscheller, J., \& Rupp, R. (2005). EEG-based asynchronous BCl controls functional electrical stimulation in a tetraplegic patient. EURASIP Journal on Applied Signal Processing, 2005, 3152-3155. 
Phillips-Silver, J., \& Trainor, L. J. (2005). Feeling the beat: movement influences infant rhythm perception. Science, 308(5727), 1430-1430.

Pogosyan, A., Gaynor, L. D., Eusebio, A., \& Brown, P. (2009). Boosting Cortical Activity at Beta-Band Frequencies Slows Movement in Humans. Current Biology, 19(19), 1637-1641. https://doi.org/10.1016/j.cub.2009.07.074

Power, A. J., Mead, N., Barnes, L., \& Goswami, U. (2012). Neural entrainment to rhythmically presented auditory, visual, and audio-visual speech in children. Frontiers in Psychology, 3. Retrieved from https://www.ncbi.nlm.nih.gov/pmc/articles/PMC3400256/

Power, A. J., Mead, N., Barnes, L., \& Goswami, U. (2013). Neural entrainment to rhythmic speech in children with developmental dyslexia. Frontiers in Human Neuroscience, 7. Retrieved from https://www.ncbi.nlm.nih.gov/pmc/articles/PMC3842021/

Regan, D. (1989). Human brain electrophysiology: evoked potentials and evoked magnetic fields in science and medicine. Retrieved from http://www.citeulike.org/group/8861/article/4033861

Repp, B. H. (2005). Sensorimotor synchronization: A review of the tapping literature. Psychonomic Bulletin \& Review, 12(6), 969-992.

Repp, B. H. (2010). Sensorimotor synchronization and perception of timing: effects of music training and task experience. Human Movement Science, 29(2), 200-213.

Repp, B. H., \& Su, Y.-H. (2013). Sensorimotor synchronization: A review of recent research (2006-2012). Psychonomic Bulletin \& Review, 20(3), 403-452. https://doi.org/10.3758/s13423-012-0371-2

Schlaug, G., Norton, A., Overy, K., \& Winner, E. (2005). Effects of music training on the child's brain and cognitive development. Annals of the New York Academy of Sciences, 1060(1), 219-230.

Schroeder, C. E., \& Lakatos, P. (2009). Low-frequency neuronal oscillations as instruments of sensory selection. Trends in Neurosciences, 32(1), 9-18. https://doi.org/10.1016/j.tins.2008.09.012 
Shahin, A. J., Trainor, L. J., Roberts, L. E., Backer, K. C., \& Miller, L. M. (2010). Development of auditory phase-locked activity for music sounds. Journal of Neurophysiology, 103(1), 218-229.

Snyder, J. S., \& Large, E. W. (2005). Gamma-band activity reflects the metric structure of rhythmic tone sequences. Cognitive Brain Research, 24(1), 117-126.

Stefanics, G., Háden, G., Huotilainen, M., Balázs, L., Sziller, I., Beke, A., ... Winkler, I. (2007). Auditory temporal grouping in newborn infants. Psychophysiology, 44(5), 697-702. https://doi.org/10.1111/j.1469-8986.2007.00540.x

Stefanics, G., Hangya, B., Hernádi, I., Winkler, I., Lakatos, P., \& Ulbert, I. (2010). Phase entrainment of human delta oscillations can mediate the effects of expectation on reaction speed. The Journal of Neuroscience : The Official Journal of the Society for Neuroscience, 30(41), 13578-13585.

Stupacher, J., Wood, G., \& Witte, M. (2017). Neural Entrainment to Polyrhythms: A Comparison of Musicians and Non-musicians. Frontiers in Neuroscience, 11. https://doi.org/10.3389/fnins.2017.00208

Takano, T., \& Ogawa, T. (1998). Characterization of developmental changes in EEG-gamma band activity during childhood using the autoregressive model. Pediatrics International, 40(5), 446-452. https://doi.org/10.1111/j.1442-200X.1998.tb01966.x

Tallal, P., \& Gaab, N. (2006). Dynamic auditory processing, musical experience and language development. Trends in Neurosciences, 29(7), 382-390.

Thomson, J. M., \& Goswami, U. (2008). Rhythmic processing in children with developmental dyslexia: auditory and motor rhythms link to reading and spelling. Journal of Physiology-Paris, 102(1), 120-129.

Thut, G., Schyns, P. G., \& Gross, J. (2011). Entrainment of Perceptually Relevant Brain Oscillations by Non-Invasive Rhythmic Stimulation of the Human Brain. Frontiers in Psychology, 2. https://doi.org/10.3389/fpsyg.2011.00170 
Tierney, A., \& Kraus, N. (2014a). Auditory-motor entrainment and phonological skills: precise auditory timing hypothesis (PATH). Frontiers in Human Neuroscience, 8, 949.

Tierney, A., \& Kraus, N. (2014b). Neural entrainment to the rhythmic structure of music. Journal of Cognitive Neuroscience. Retrieved from http://www.mitpressjournals.org/doi/abs/10.1162/jocn_a_00704

Tierney, A. T., \& Kraus, N. (2013). The ability to tap to a beat relates to cognitive, linguistic, and perceptual skills. Brain and Language, 124(3), 225-231.

Tierney, A. T., Krizman, J., \& Kraus, N. (2015). Music training alters the course of adolescent auditory development. Proceedings of the National Academy of Sciences, 112(32), 10062-10067.

Tóth, D., Csépe, V., Vaessen, A., \& Blomert, L. (2014). 3DM-H: A diszlexia differenciáldiagnózisa. Az olvasás és helyesírás kognitív elemzése. Technikai kézikönyv.[3DM-H: Dyslexia differential diagnosis. Cognitive analysis of reading and spelling. Technical manual.]. Nyíregyháza, Hungary: Kogentum.

Ward, L. M. (2003). Synchronous neural oscillations and cognitive processes. Trends in Cognitive Sciences, 7(12), 553-559.

Winkler, I., Háden, G. P., Ladinig, O., Sziller, I., \& Honing, H. (2009). Newborn infants detect the beat in music. Proceedings of the National Academy of Sciences, 106(7), 2468-2471. https://doi.org/10.1073/pnas.0809035106

Zatorre, R. J., Chen, J. L., \& Penhune, V. B. (2007). When the brain plays music: auditory-motor interactions in music perception and production. Nature Reviews. Neuroscience, 8(7), 547.

Zion Golumbic, E. M., Ding, N., Bickel, S., Lakatos, P., Schevon, C. A., McKhann, G. M., ... Schroeder, C. E. (2013). Mechanisms Underlying Selective Neuronal Tracking of Attended Speech at a "Cocktail Party." Neuron, 77(5), 980-991. https://doi.org/10.1016/j.neuron.2012.12.037 